\title{
Production Potential of Buckwheat (Fagopyrum esculentum Moench) as Influenced by Genotypes and Fertilizer Levels in Northern Transition Zone of Karnataka, India
}

\author{
Maruti", U.K. Hulihalli and B.N. Aravind Kumar \\ Department of Agronomy, College of Agriculture, Dharwad, University of Agricultural \\ Sciences, Dharwad-580005, Karnataka, India \\ *Corresponding author
}

\section{Keywords}

Buckwheat, LAI, SPAD values, Seeds per cluster

Article Info

Accepted:

06 August 2018

Available Online:

10 September 2018

\section{A B S T R A C T}

A field experiment was conducted to know the production potential of buckwheat (Fagopyrum esculentum Moench) as influenced by genotypes and fertilizer levels in Northern Transition Zone of Karnataka was carried out at Main Agriculture Research Station, University of Agricultural Sciences, Dharwad, Karnataka during kharif 2017. The experiment was laid out in split plot design with three replications comprising of twentyfour treatment combinations. The treatment includes two genotypes viz., $\mathrm{G}_{1}$ : IC-79147 and $\mathrm{G}_{2}$ : PRB-1 as main plots with twelve fertilizer levels $\left(\mathrm{N}: \mathrm{P}_{2} \mathrm{O}_{5}: \mathrm{K}_{2} \mathrm{O} \mathrm{kg} \mathrm{ha}{ }^{-1}\right)$ viz., $\mathrm{F}_{1}: 20$ : 10: $0, \mathrm{~F}_{2}: 20: 10: 10, \mathrm{~F}_{3}: 20: 20: 0, \mathrm{~F}_{4}: 20: 20: 10, \mathrm{~F}_{5}: 40: 10: 0, \mathrm{~F}_{6}: 40: 10: 10, \mathrm{~F}_{7}: 40: 20:$ 0, $\mathrm{F}_{8}: 40: 20: 10, \mathrm{~F}_{9}: 60: 10: 0, \mathrm{~F}_{10}: 60: 10: 10, \mathrm{~F}_{11}: 60: 20: 0, \mathrm{~F}_{12}: 60: 20: 10$ as subplots. The results revealed that genotype IC-79147 recorded significantly higher seed yield (8.07 $\mathrm{q} \mathrm{ha}{ }^{-1}$ ). Among the fertilizer levels, application of 60: 20: $10 \mathrm{~kg} \mathrm{~N}: \mathrm{P}_{2} \mathrm{O}_{5}: \mathrm{K}_{2} \mathrm{O} \mathrm{kg} \mathrm{ha}{ }^{-1}$ was recorded significantly higher seed $\left(8.47 \mathrm{q} \mathrm{ha}^{-1}\right)$ and straw yield $\left(19.23 \mathrm{q} \mathrm{ha}^{-1}\right)$. The genotype IC-79147 with application of 60: 20: $10 \mathrm{~kg} \mathrm{~N}: \mathrm{P}_{2} \mathrm{O}_{5}: \mathrm{K}_{2} \mathrm{O} \mathrm{kg} \mathrm{ha} \mathrm{kg}^{-1}$ recorded significantly higher seed yield $\left(9.43 \mathrm{q} \mathrm{ha}^{-1}\right)$ and yield attributes viz., number of clusters per plant (7.21), seeds per cluster (10.17), 1000 seed weight $(25.71 \mathrm{~g}$ ), as well as net returns (₹ $30,090 \mathrm{ha}^{-1}$ ) and $\mathrm{BC}$ ratio (3.11), which was on par with application of $\mathrm{F}_{10}$ and $\mathrm{F}_{11}$ fertilizer levels along with IC-79147 genotype. PRB-1 genotype with 60: 20: $10 \mathrm{~kg} \mathrm{~N}: \mathrm{P}_{2} \mathrm{O}_{5}: \mathrm{K}_{2} \mathrm{O} \mathrm{kg}$ $\mathrm{ha}^{-1}$ fertility level recorded significantly higher straw yield $\left(17.01 \mathrm{q} \mathrm{ha}^{-1}\right)$ and growth attributes viz., plant height $(102.6 \mathrm{~cm})$, total dry matter production per plant $(8.55 \mathrm{~g})$, LAI (2.15) and number of branches per plant (9.64) at harvest, which was on par with PRB-1 genotype along with $\mathrm{F}_{10}$ and $\mathrm{F}_{11}$ fertility levels as compared to other interactions.

\section{Introduction}

Common buckwheat (Fagopyrum esculentum Moench) is herbaceous erect annual plant with diploid chromosome number $(2 \mathrm{n}=16)$. It belongs to the family polygonaceae. Buckwheat is one of the most important pseudo cereal crops of the mountain region widely cultivated in the middle and higher Himalayas between 1800 to $4500 \mathrm{~m}$ from MSL during kharif season. Buckwheat is originated in temperate Central Asia. Buckwheat is mainly cultivated in the temperate zones of the Northern hemisphere 
(Oshini, 2004), especially in Russia. It is also grown in USA, Canada, France, Germany, U.K., Denmark, Poland, Holland, Sweden, Australia, Bulgaria, Romania, Italy, Japan, South Africa, Brazil, China, South Korea, Nepal and Bhutan.

In India, it is a traditional crop of the high altitudes of Himalayan region having multifarious utility. It is cultivated by the poor farmers as a food grain crop and ensured livelihood and nutritional security of thousands of marginal farmers residing in difficult and remote areas far away from assured public food distribution system and it is known by its various vernacular names such as ogal, phaphar and kuttu.

Production and management of every crop, genotype and fertilizer levels are play very important role. Every genotype has its own capacity for better-use of nutrient, light, temperature, precipitation and other production factors. Fertilizer levels are very important in buckwheat for better crop growth and translocation of photosynthates from source to sink which is very important for the development of economic part and to achieve higher yield by producing more branches, because it does not have tillering capacity as like other cereals. Buckwheat is highly responsive to applied and initial nitrogen and phosphorus content of the soil.

\section{Materials and Methods}

The field experiment on growth and development of buckwheat (Fagopyrum esculentum Moench) as influenced by genotypes and fertilizer levels was carried out at Main Agriculture Research Station, Dharwad, Karnataka during kharif 2017. The experimental field is located between at $15^{\circ} 29^{\prime} \mathrm{N}$ latitude, $74^{0} 59^{\prime} \mathrm{E}$ longitude at an altitude of $689 \mathrm{~m}$ above mean sea level and it comes under Northern Transition Agro- climatic Zone (Zone-8) of Karnataka. The experiment was laid in split-plot design with three replications comprising of twenty-four treatment combinations. The treatments include two genotypes viz., $\mathrm{G}_{1}$ : IC-79147 and $\mathrm{G}_{2}$ : PRB-1 as main plots with twelve fertilizer levels $\left(\mathrm{N}: \mathrm{P}_{2} \mathrm{O}_{5}: \mathrm{K}_{2} \mathrm{O} \mathrm{kg} \mathrm{ha}{ }^{-1}\right.$ ) viz., $\mathrm{F}_{1}:$ 20: 10: $0, \mathrm{~F}_{2}: 20: 10: 10, \mathrm{~F}_{3}: 20: 20: 0, \mathrm{~F}_{4}: 20: 20: 10$, $\mathrm{F}_{5}: 40: 10: 0, \mathrm{~F}_{6}: 40: 10: 10, \mathrm{~F}_{7}: 40: 20: 0, \mathrm{~F}_{8}$ : 40: 20: 10, F9: 60: 10: 0, F $10: 60: 10: 10, \mathrm{~F}_{11}$ : 60: 20: 0, $\mathrm{F}_{12}:$ 60: 20: 10 as subplots. Soil of the experimental site was vertisols with soil $\mathrm{pH} 7.59$ and its nutrient status was low in available nitrogen $\left(226.35 \mathrm{~kg} \mathrm{ha}^{-1}\right)$, medium in available phosphorus (29.52 $\left.\mathrm{kg} \mathrm{ha}^{-1}\right)$ and high with available potassium $\left(371.3 \mathrm{~kg} \mathrm{ha}^{-1}\right)$. The standard procedures are followed to record the growth and yield observations. Leaf Area Index (LAI) was calculated by disc method as suggested by Sestak et al., (1971).

\section{Results and Discussion}

\section{Growth parameters}

The data on growth attributes viz., plant height, number of leaves per plant, number of branches per plant, leaf area, leaf area index, total dry matter production per plant at harvest as influenced by buckwheat genotypes, fertility levels and their interaction effects are presented in Table 1 and 2.

The genotype PRB-1 recorded significantly higher plant height $(102.6 \mathrm{~cm})$, number of leaves per plant (27.32), number of branches per plant (9.64), leaf area $\left(6.45 \mathrm{dm}^{2}\right.$ plant $\left.^{-1}\right)$, leaf area index (2.15) and total dry matter production per plant $(8.55 \mathrm{~g})$ as compared to IC-79147 $\left(70.0 \mathrm{~cm}, 19.71,6.69,3.57 \mathrm{dm}^{2}\right.$ plant $^{-1}, 1.19$ and $6.85 \mathrm{~g}$ respectively) at harvest stage of buckwheat. Among the fertility levels, application of 60: 20: $10 \mathrm{~N}: \mathrm{P}_{2} \mathrm{O}_{5}: \mathrm{K}_{2} \mathrm{O} \mathrm{kg} \mathrm{ha}{ }^{-1}$ was recorded significantly higher plant height $(91.1 \mathrm{~cm})$, number of leaves per plant $(28.38)$, number of branches per plant (10.58), leaf 
area $\left(5.76 \mathrm{dm}^{2}\right.$ plant $\left.^{-1}\right)$, leaf area index (1.92) and total dry matter production per plant $(9.57$ g) and which was on par with fertility levels $F_{11}, \quad F_{10}$ and $F_{9}$ with respect all above mentioned growth attributes at harvest as compared to other fertility levels.

Interaction effects between genotypes and fertility levels were found significantly differed with respect all growth attributes of buckwheat. The genotype PRB-1 recorded significantly higher growth attributes viz., plant height $(106.7 \mathrm{~cm})$, number of leaves per plant (34.03), number of branches per plant (12.16), leaf area $\left(7.16 \mathrm{dm}^{2}\right.$ plant $\left.^{-1}\right)$, leaf area index (2.39) and total dry matter production per plant $(10.44 \mathrm{~g})$ along with application of $\mathrm{N}: \mathrm{P}_{2} \mathrm{O}_{5}: \mathrm{K}_{2} \mathrm{O}$ at 60: 20: $10 \mathrm{~kg} \mathrm{ha}^{-1}$, Which was found on par with interaction effect of $\mathrm{G}_{2} \times \mathrm{F}_{11}$ and $\mathrm{G}_{2} \times \mathrm{F}_{10}$. The lower growth attributes were recorded with $\mathrm{G}_{1} \times \mathrm{F}_{1}$ interaction. Higher growth attributes with PRB-1 and fertility level of 60: 20: $10 \mathrm{~N}: \mathrm{P}_{2} \mathrm{O}_{5}: \mathrm{K}_{2} \mathrm{O} \mathrm{kg} \mathrm{ha}{ }^{-1}$ might be due the genotype (PRB-1) characters and long vegetative period (90 days) and it was highly responsive to the applied and available resources like moisture, nutrients, sunlight contributing higher plant height, number of leaves and branches per plant, leaf area, leaf area index and total dry matter production per plant as compared to IC-79147 which matures early (70 days). Similar results were reprted by Hulihalli and Shanthaveerayya (2017) and Christensen et al., (2007),

\section{Yield parameters}

The data on yield attributes as influenced by genotypes, fertilizer levels and their interaction effects are presented in Table 3.

Significantly higher yield attributes viz., number of clusters per plant (7.21), number of seeds per cluster (10.17) and 1000 seed weight $(25.71 \mathrm{~g})$ were recorded with IC-79147 genotype as compared to PRB-1 (4.76, 5.79 and $19.97 \mathrm{~g}$ respectively). Among the fertility levels application of 60: 20: $10 \mathrm{~N}: \mathrm{P}_{2} \mathrm{O}_{5}: \mathrm{K}_{2} \mathrm{O}$ $\mathrm{kg} \mathrm{ha}^{-1}$ was recorded significantly higher yield attributes viz., number of clusters per plant (8.79), number of seeds per cluster (10.53) and 1000 seed weight $(27.23 \mathrm{~g})$ over the other fertility levels. However, it was found on par with $F_{11}, F_{10}$ and $F_{9}$ fertilizer levels with respect to all yield attributes.

Among the interaction effects IC-79147 along with application of 60: 20: $10 \mathrm{~N}: \mathrm{P}_{2} \mathrm{O}_{5}: \mathrm{K}_{2} \mathrm{O}$ $\mathrm{kg} \mathrm{ha}^{-1}$ was recorded significantly higher yield attributes namely number of clusters per plant (10.88), number of seeds per cluster (13.63) and 1000 seed weight (30.02 g), which was found on par with interactions of $\mathrm{G} 1 \times \mathrm{F}_{11}$ and $\mathrm{G} 1 \times \mathrm{F}_{10}$. The increase in yield attributes may be due to better utilization of available resources and nitrogen which may involve in energy transformations, activation of enzymes in carbohydrate metabolism and consequently greater transloacation and accumuation of photosynthates into reproductive parts and also due to genetic potentiality of the genotype (IC-79147).

This may also attributed to seeds IC-79147 were larger and bolder in size as compared to PRB-1. Similar results were reported by Mahata (2016), Hongmei et al., (2003) and Warchoowa et al., (1991)

\section{Seed yield, straw yield and harvest index}

The data on seed yield, straw yield and harvest index as affected by genotypes, fertilizer levels and their interaction effects are presented in Table 4.

Significantly higher seed yield and harvest index was recorded with IC-79147 genotype (8.07 $\mathrm{q} \mathrm{ha}^{-1}$ and 38.43 respectively) than PRB$1\left(6.13 \mathrm{q} \mathrm{ha}^{-1}\right.$ and 26.76), whereas PRB-1 registered with higher straw yield (17.01 q $\left.\mathrm{ha}^{-1}\right)$ as compared to IC-79147 (13.11 q ha $\left.{ }^{-1}\right)$. 
Table.1 Growth attributes of buckwheat as influenced by genotypes, fertilizer levels and their interaction effects

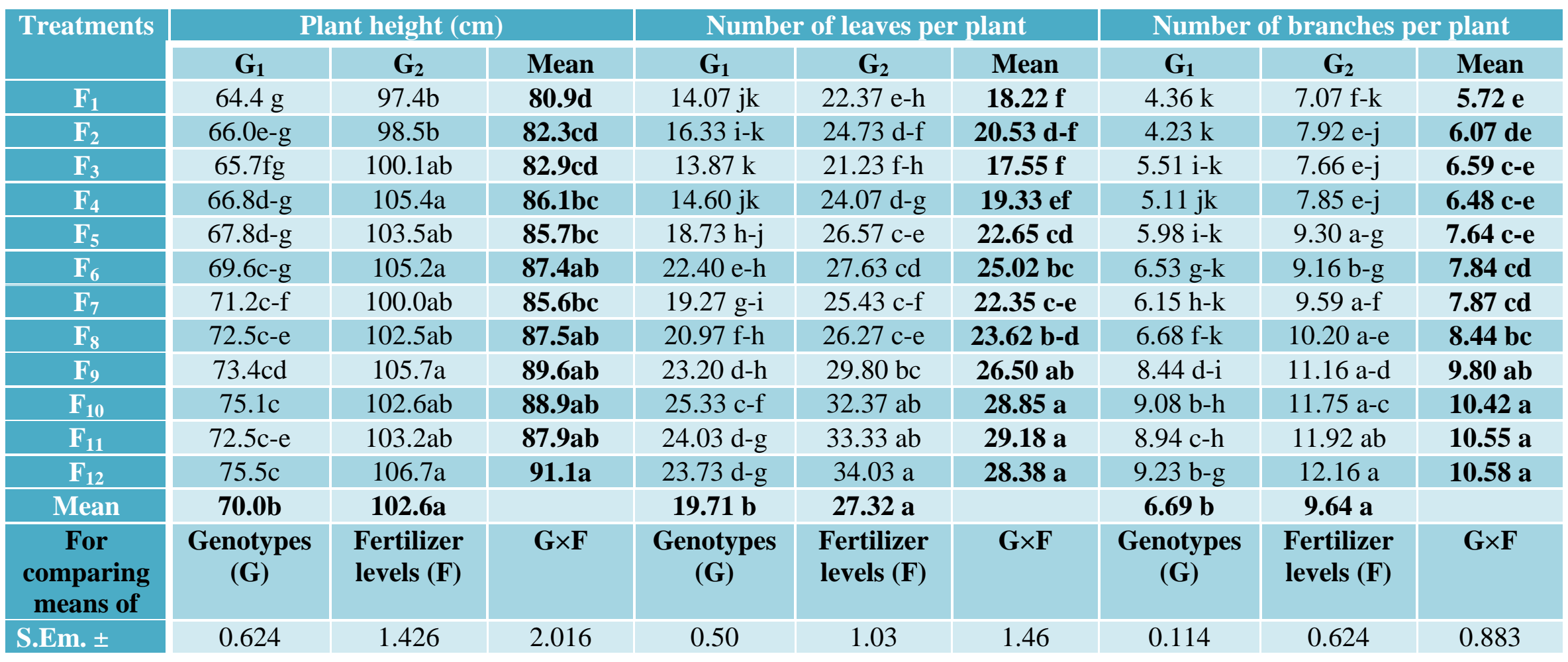

\begin{tabular}{|c|c|c|}
\hline G: Genotypes & \multicolumn{2}{|c|}{ F: Fertilizer levels $\left(\mathrm{kg} \mathrm{ha}^{-1}\right)$} \\
\hline $\mathrm{G}_{1}: \mathrm{IC}-79147$ & $\mathbf{F}_{1}: \mathrm{N}_{1} \mathrm{P}_{1} \mathrm{~K}_{1}(20: 10: 0)$ & $\mathbf{F}_{7}: \mathrm{N}_{2} \mathrm{P}_{2} \mathrm{~K}_{1}(40: 20: 0)$ \\
\hline \multirow[t]{5}{*}{$\mathrm{G}_{2}:$ PRB-1 } & $\mathbf{F}_{2}: \mathrm{N}_{1} \mathrm{P}_{1} \mathrm{~K}_{2}(20: 10: 10$ & $\mathbf{F}_{\mathbf{8}}: \mathrm{N}_{2} \mathrm{P}_{2} \mathrm{~K}_{2}(40: 20: 10)$ \\
\hline & $\mathbf{F}_{3}: \mathrm{N}_{1} \mathrm{P}_{2} \mathrm{~K}_{1}(20: 20: 0)$ & $\mathbf{F}_{9}: \mathrm{N}_{3} \mathrm{P}_{1} \mathrm{~K}_{1}(60: 10: 0)$ \\
\hline & $\mathbf{F}_{4}: \mathrm{N}_{1} \mathrm{P}_{2} \mathrm{~K}_{2}(20: 20: 10)$ & $\mathbf{F}_{10}: \mathrm{N}_{3} \mathrm{P}_{1} \mathrm{~K}_{2}(60: 10: 10)$ \\
\hline & $\mathbf{F}_{5}: \mathrm{N}_{2} \mathrm{P}_{1} \mathrm{~K}_{1}(40: 10: 0)$ & $\mathbf{F}_{11}: \mathrm{N}_{3} \mathrm{P}_{2} \mathrm{~K}_{1}(60: 20: 0)$ \\
\hline & $\mathbf{F}_{6}: \mathrm{N}_{2} \mathrm{P}_{1} \mathrm{~K}_{2}(40: 10: 10)$ & $\mathbf{F}_{12}: \mathrm{N}_{3} \mathrm{P}_{2} \mathrm{~K}_{2}(60: 20: 10)$ \\
\hline
\end{tabular}


Table.2 Growth attributes of buckwheat as influenced by genotypes, fertilizer levels and their interaction effects

\begin{tabular}{|c|c|c|c|c|c|c|c|c|c|}
\hline \multirow[t]{2}{*}{ Treatments } & \multicolumn{3}{|c|}{ Leaf area $\left(\mathrm{dm}^{2}\right.$ plant $\left.^{-1}\right)$} & \multicolumn{3}{|c|}{ Leaf area index } & \multicolumn{3}{|c|}{ Total dry matter (g plant $\left.{ }^{-1}\right)$} \\
\hline & $\mathbf{G}_{1}$ & $\mathbf{G}_{2}$ & Mean & $\mathbf{G}_{1}$ & $\mathbf{G}_{2}$ & Mean & $\mathbf{G}_{1}$ & $\mathbf{G}_{2}$ & Mean \\
\hline $\mathbf{F}_{1}$ & $2.80 \mathrm{i}$ & $5.78 \mathrm{~d}$ & $4.29 \mathrm{e}$ & $0.93 \mathrm{i}$ & $1.93 \mathrm{~d}$ & $1.43 \mathrm{~d}$ & $5.34 \mathrm{~g}$ & $6.95 \mathrm{c}-\mathrm{g}$ & $6.15 \mathrm{~d}$ \\
\hline $\mathbf{F}_{2}$ & $2.85 \mathrm{i}$ & $5.86 \mathrm{~d}$ & $4.36 \mathrm{e}$ & $0.95 \mathrm{i}$ & $1.95 \mathrm{~d}$ & $1.45 \mathrm{~d}$ & $6.08 \mathrm{e}-\mathrm{g}$ & $7.08 \mathrm{c}-\mathrm{g}$ & $6.58 \mathrm{~d}$ \\
\hline$F_{3}$ & $2.79 \mathrm{i}$ & $5.71 \mathrm{~d}$ & $4.25 \mathrm{e}$ & $0.93 \mathrm{i}$ & $1.90 \mathrm{~d}$ & $1.42 \mathrm{~d}$ & $5.97 \mathrm{fg}$ & $7.02 \mathrm{c}-\mathrm{g}$ & $6.50 \mathrm{~d}$ \\
\hline $\mathbf{F}_{4}$ & $2.87 \mathrm{i}$ & $5.85 \mathrm{~d}$ & $4.36 \mathrm{e}$ & $0.96 \mathrm{i}$ & $1.95 \mathrm{~d}$ & $1.45 \mathrm{~d}$ & $5.64 \mathrm{~g}$ & $7.31 \mathrm{c}-\mathrm{g}$ & $6.48 \mathrm{~d}$ \\
\hline $\mathrm{F}_{5}$ & $3.43 \mathrm{~h}$ & $6.38 \mathrm{c}$ & $4.91 \mathrm{~d}$ & $1.14 \mathrm{~h}$ & $2.13 \mathrm{c}$ & $1.64 \mathrm{c}$ & $6.31 \mathrm{~d}-\mathrm{g}$ & $8.12 b-f$ & $7.22 \mathrm{~d}$ \\
\hline $\mathrm{F}_{6}$ & $3.57 \mathrm{gh}$ & $6.55 \mathrm{bc}$ & $5.06 \mathrm{c}$ & $1.19 \mathrm{gh}$ & $2.18 \mathrm{bc}$ & $1.69 \mathrm{bc}$ & $6.52 \mathrm{c}-\mathrm{g}$ & $8.41 \mathrm{a}-\mathrm{d}$ & $7.47 \mathrm{~cd}$ \\
\hline$F_{7}$ & $3.64 \mathrm{~g}$ & $6.41 \mathrm{c}$ & $5.03 \mathrm{c}$ & $1.21 \mathrm{~g}$ & $2.14 \mathrm{c}$ & $1.68 \mathrm{bc}$ & $6.70 \mathrm{c}-\mathrm{g}$ & $8.64 \mathrm{a}-\mathrm{c}$ & $7.67 \mathrm{~b}-\mathrm{d}$ \\
\hline $\mathrm{F}_{8}$ & $3.68 \mathrm{~g}$ & $6.62 \mathrm{~b}$ & $5.15 \mathrm{c}$ & $1.23 \mathrm{~g}$ & $2.21 \mathrm{~b}$ & $1.72 \mathrm{~b}$ & $7.06 \mathrm{c}-\mathrm{g}$ & 8.35 a-e & $7.71 \mathrm{~b}-\mathrm{d}$ \\
\hline$F_{9}$ & $4.18 \mathrm{f}$ & $7.06 \mathrm{a}$ & $5.62 \mathrm{~b}$ & $1.39 \mathrm{f}$ & $2.35 \mathrm{a}$ & 1.87 a & $7.38 \mathrm{c}-\mathrm{g}$ & $10.15 \mathrm{ab}$ & 8.76 a-c \\
\hline$F_{10}$ & 4.25 ef & $7.00 \mathrm{a}$ & $5.63 \mathrm{~b}$ & $1.42 \mathrm{ef}$ & $2.33 \mathrm{a}$ & 1.88 a & $8.04 b-f$ & $10.01 \mathrm{ab}$ & $9.02 \mathrm{ab}$ \\
\hline$F_{11}$ & $4.39 \mathrm{e}$ & 7.07a & $5.73 \mathrm{ab}$ & $1.45 \mathrm{e}$ & $2.36 \mathrm{a}$ & $1.91 \mathrm{a}$ & $8.52 \mathrm{a}-\mathrm{d}$ & $10.13 \mathrm{ab}$ & $9.32 \mathrm{a}$ \\
\hline $\mathbf{F}_{12}$ & $4.36 \mathrm{e}$ & $7.16 \mathrm{a}$ & $5.76 \mathrm{a}$ & $1.46 \mathrm{e}$ & $2.39 \mathrm{a}$ & $1.92 \mathrm{a}$ & $8.69 \mathrm{a}-\mathrm{c}$ & $10.44 \mathrm{a}$ & $9.57 \mathbf{a}$ \\
\hline Mean & $3.57 \mathrm{~b}$ & $6.45 \mathrm{a}$ & & $1.19 \mathrm{~b}$ & $2.15 \mathrm{a}$ & & $6.85 \mathrm{~b}$ & $8.55 \mathrm{a}$ & \\
\hline $\begin{array}{c}\text { For } \\
\text { comparing } \\
\text { means of }\end{array}$ & $\begin{array}{c}\text { Genotypes } \\
\text { (G) }\end{array}$ & $\begin{array}{l}\text { Fertilizer } \\
\text { levels (F) }\end{array}$ & $\mathbf{G} \times \mathbf{F}$ & $\begin{array}{c}\text { Genotypes } \\
\text { (G) }\end{array}$ & $\begin{array}{l}\text { Fertilizer } \\
\text { levels (F) }\end{array}$ & $\mathbf{G} \times \mathbf{F}$ & $\begin{array}{c}\text { Genotypes } \\
\text { (G) }\end{array}$ & $\begin{array}{l}\text { Fertilizer } \\
\text { levels (F) }\end{array}$ & $\mathbf{G} \times \mathbf{F}$ \\
\hline S.Em. \pm & 0.0079 & 0.042 & 0.059 & 0.003 & 0.014 & 0.020 & 0.092 & 0.481 & 0.680 \\
\hline
\end{tabular}

\begin{tabular}{|l|}
\hline G: Genotypes \\
\hline $\mathrm{G}_{1}$ : IC-79147 \\
\hline $\mathrm{G}_{2}$ : PRB-1 \\
\hline \\
\hline
\end{tabular}

\begin{tabular}{l}
$F_{1}: N_{1} P_{1} K_{1}(20: 10: 0)$ \\
$F_{2}: N_{1} P_{1} K_{2}(20: 10: 10$ \\
$F_{3}: N_{1} P_{2} K_{1}(20: 20: 0)$ \\
$F_{4}: N_{1} P_{2} K_{2}(20: 20: 10)$ \\
$F_{5}: N_{2} P_{1} K_{1}(40: 10: 0)$ \\
$F_{6}: N_{2} P_{1} K_{2}(40: 10: 10)$ \\
\hline
\end{tabular}

F: Fertilizer levels $\left(\mathrm{kg} \mathrm{ha}^{-1}\right)$ 
Table.3 Number clusters per plant, seeds per cluster and test weight of buckwheat as influenced by genotypes, fertilizer levels and their interaction effects

\begin{tabular}{|c|c|c|c|c|c|c|c|c|c|}
\hline \multirow[t]{2}{*}{ Treatments } & \multicolumn{3}{|c|}{ Number of clusters plant ${ }^{-1}$} & \multicolumn{3}{|c|}{ Seeds cluster $^{-1}$} & \multicolumn{3}{|c|}{ Test weight (1000 seeds, g) } \\
\hline & $\mathbf{G}_{1}$ & $\mathbf{G}_{2}$ & Mean & $\mathbf{G}_{1}$ & $\mathbf{G}_{2}$ & Mean & $\mathbf{G}_{1}$ & $\mathbf{G}_{2}$ & Mean \\
\hline $\mathbf{F}_{1}$ & $5.14 \mathrm{e}-\mathrm{h}$ & $2.80 \mathrm{i}$ & $3.97 f$ & $5.14 \mathrm{e}-\mathrm{h}$ & $2.80 \mathrm{i}$ & $3.97 f$ & $5.14 \mathrm{e}-\mathrm{h}$ & $2.80 \mathrm{i}$ & $3.97 f$ \\
\hline $\mathrm{F}_{2}$ & $4.92 f-h$ & 3.60hi & $4.26 f$ & $4.92 f-h$ & 3.60hi & $4.26 f$ & $4.92 f-h$ & 3.60hi & $4.26 f$ \\
\hline $\mathbf{F}_{3}$ & 3.68hi & $4.57 \mathrm{~g}-\mathrm{i}$ & $4.12 f$ & 3.68hi & $4.57 \mathrm{~g}-\mathrm{i}$ & $4.12 f$ & 3.68hi & $4.57 \mathrm{~g}-\mathrm{i}$ & $4.12 f$ \\
\hline $\mathbf{F}_{4}$ & $5.30 \mathrm{~d}-\mathrm{h}$ & 3.66hi & 4.48ef & $5.30 \mathrm{~d}-\mathrm{h}$ & 3.66hi & 4.48ef & $5.30 \mathrm{~d}-\mathrm{h}$ & 3.66hi & 4.48ef \\
\hline$F_{5}$ & $6.96 c-f$ & $4.62 \mathrm{~g}-\mathrm{i}$ & 5.79de & $6.96 c-f$ & $4.62 \mathrm{~g}-\mathrm{i}$ & 5.79de & $6.96 c-f$ & $4.62 \mathrm{~g}-\mathrm{i}$ & 5.79de \\
\hline $\mathrm{F}_{6}$ & $7.15 \mathrm{c}-\mathrm{e}$ & 3.54hi & $5.35 \mathrm{~d}-\mathrm{f}$ & $7.15 \mathrm{c}-\mathrm{e}$ & 3.54hi & $5.35 \mathrm{~d}-\mathrm{f}$ & $7.15 \mathrm{c}-\mathrm{e}$ & 3.54hi & $5.35 \mathrm{~d}-\mathrm{f}$ \\
\hline$F_{7}$ & $6.83 c-f$ & $5.31 \mathrm{~d}-\mathrm{h}$ & 6.07cd & $6.83 c-f$ & $5.31 \mathrm{~d}-\mathrm{h}$ & $6.07 \mathrm{~cd}$ & $6.83 c-f$ & 5.31d-h & 6.07cd \\
\hline $\mathrm{F}_{8}$ & $7.28 \mathrm{~cd}$ & $4.64 \mathrm{~g}-\mathrm{i}$ & $5.96 \mathrm{~cd}$ & $7.28 \mathrm{~cd}$ & $4.64 \mathrm{~g}-\mathrm{i}$ & $5.96 \mathrm{~cd}$ & $7.28 \mathrm{~cd}$ & $4.64 \mathrm{~g}-\mathrm{i}$ & $5.96 \mathrm{~cd}$ \\
\hline $\mathrm{F}_{9}$ & $9.15 \mathrm{ab}$ & $6.01 \mathrm{~d}-\mathrm{g}$ & 7.58ab & $9.15 \mathrm{ab}$ & $6.01 \mathrm{~d}-\mathrm{g}$ & 7.58ab & $9.15 \mathrm{ab}$ & $6.01 \mathrm{~d}-\mathrm{g}$ & 7.58ab \\
\hline$F_{10}$ & $8.75 b c$ & $5.80 \mathrm{~d}-\mathrm{g}$ & $7.27 b c$ & $8.75 b c$ & $5.80 \mathrm{~d}-\mathrm{g}$ & $7.27 b c$ & $8.75 b c$ & $5.80 \mathrm{~d}-\mathrm{g}$ & $7.27 b c$ \\
\hline$F_{11}$ & $10.45 a b$ & $5.82 \mathrm{~d}-\mathrm{g}$ & 8.14ab & $10.45 a b$ & $5.82 \mathrm{~d}-\mathrm{g}$ & 8.14ab & $10.45 a b$ & $5.82 \mathrm{~d}-\mathrm{g}$ & 8.14ab \\
\hline$F_{12}$ & $10.88 \mathrm{a}$ & $6.70 \mathrm{~d}-\mathrm{f}$ & $8.79 \mathrm{a}$ & $10.88 \mathrm{a}$ & $6.70 \mathrm{~d}-\mathrm{f}$ & $8.79 \mathrm{a}$ & $10.88 \mathrm{a}$ & $6.70 \mathrm{~d}-\mathrm{f}$ & 8.79a \\
\hline Mean & $7.21 \mathrm{a}$ & $4.76 \mathrm{~b}$ & & $7.21 \mathrm{a}$ & $4.76 \mathrm{~b}$ & & $7.21 \mathrm{a}$ & $4.76 \mathrm{~b}$ & \\
\hline $\begin{array}{c}\text { For } \\
\text { comparing } \\
\text { means of }\end{array}$ & $\begin{array}{c}\text { Genotypes } \\
\text { (G) }\end{array}$ & $\begin{array}{l}\text { Fertilizer } \\
\text { levels (F) }\end{array}$ & $\mathbf{G} \times \mathbf{F}$ & $\begin{array}{c}\text { Genotypes } \\
\text { (G) }\end{array}$ & $\begin{array}{l}\text { Fertilizer } \\
\text { levels ( } F)\end{array}$ & $\mathbf{G} \times \mathbf{F}$ & $\begin{array}{c}\text { Genotypes } \\
\text { (G) }\end{array}$ & $\begin{array}{l}\text { Fertilizer } \\
\text { levels (F) }\end{array}$ & $\mathbf{G} \times \mathbf{F}$ \\
\hline S.Em. \pm & 0.159 & 0.466 & 0.660 & 0.279 & 0.801 & 1.132 & 0.379 & 0.916 & 1.296 \\
\hline
\end{tabular}

\begin{tabular}{|c|c|c|}
\hline G: Genotypes & \multicolumn{2}{|c|}{ F: Fertilizer levels $\left(\mathrm{kg} \mathrm{ha}^{-1}\right)$} \\
\hline $\mathrm{G}_{1}: \mathrm{IC}-\mathbf{7 9 1 4 7}$ & $\mathbf{F}_{1}: \mathrm{N}_{1} \mathrm{P}_{1} \mathrm{~K}_{1}(20: 10: 0)$ & $\mathbf{F}_{7}: \mathrm{N}_{2} \mathrm{P}_{2} \mathrm{~K}_{1}(40: 20: 0)$ \\
\hline $\mathrm{G}_{2}:$ PRB-1 & $\mathbf{F}_{2}: \mathrm{N}_{1} \mathrm{P}_{1} \mathrm{~K}_{2}(20: 10: 10$ & $\mathbf{F}_{\mathbf{8}}: \mathrm{N}_{2} \mathrm{P}_{2} \mathrm{~K}_{2}(40: 20: 10)$ \\
\hline & $\mathbf{F}_{3}: \mathrm{N}_{1} \mathrm{P}_{2} \mathrm{~K}_{1}(20: 20: 0)$ & $\mathbf{F}_{9}: \mathrm{N}_{3} \mathrm{P}_{1} \mathrm{~K}_{1}(60: 10: 0)$ \\
\hline & $\mathbf{F}_{4}: \mathrm{N}_{1} \mathrm{P}_{2} \mathrm{~K}_{2}(20: 20: 10)$ & $\mathbf{F}_{10}: \mathrm{N}_{3} \mathrm{P}_{1} \mathrm{~K}_{2}(60: 10: 10)$ \\
\hline & $\mathbf{F}_{\mathbf{5}}: \mathrm{N}_{2} \mathrm{P}_{1} \mathrm{~K}_{1}(40: 10: 0)$ & $\mathbf{F}_{11}: \mathrm{N}_{3} \mathrm{P}_{2} \mathrm{~K}_{1}(60: 20: 0)$ \\
\hline & $\mathbf{F}_{\mathbf{6}}: \mathrm{N}_{2} \mathrm{P}_{1} \mathrm{~K}_{2}(40: 10: 10)$ & $\mathbf{F}_{12}: \mathrm{N}_{3} \mathrm{P}_{2} \mathrm{~K}_{2}(60: 20: 10)$ \\
\hline
\end{tabular}


Table.4 Seed yield, straw yield and harvest index of buckwheat as influenced by genotypes, Fertilizer levels and their interaction effects

\begin{tabular}{|c|c|c|c|c|c|c|c|c|c|}
\hline \multirow[t]{2}{*}{ Treatments } & \multicolumn{3}{|c|}{ Seed yield $\left(q\right.$ ha $\left.^{-1}\right)$} & \multicolumn{3}{|c|}{ Straw yield $\left(q h^{-1}\right)$} & \multicolumn{3}{|c|}{ Harvest index } \\
\hline & $\mathbf{G}_{1}$ & $\mathbf{G}_{2}$ & Mean & $\mathbf{G}_{1}$ & $\mathbf{G}_{2}$ & Mean & $\mathbf{G}_{1}$ & $\mathbf{G}_{2}$ & Mean \\
\hline $\mathbf{F}_{1}$ & $6.33 \mathrm{~d}-\mathrm{g}$ & $4.75 \mathrm{~g}$ & $5.54 \mathrm{c}$ & $9.33 \mathrm{i}$ & $12.96 \mathrm{e}-\mathrm{h}$ & $11.15 \mathrm{~g}$ & $40.94 a$ & $26.74 \mathrm{e}-\mathrm{g}$ & 33.84a \\
\hline $\mathbf{F}_{2}$ & $6.84 \mathrm{~b}-\mathrm{g}$ & $4.92 \mathrm{fg}$ & $5.88 \mathrm{bc}$ & $10.90 \mathrm{~g}-\mathrm{i}$ & $11.92 \mathrm{f}-\mathrm{i}$ & $11.41 \mathrm{fg}$ & $39.92 \mathrm{ab}$ & $31.06 \mathrm{a}-\mathrm{g}$ & $35.49 \mathrm{a}$ \\
\hline$\overline{F_{3}}$ & $6.89 \mathrm{~b}-\mathrm{g}$ & $4.75 \mathrm{~g}$ & $5.82 \mathrm{bc}$ & $9.82 \mathrm{hi}$ & $13.51 \mathrm{~d}-\mathrm{g}$ & $11.66 \mathrm{fg}$ & $41.35 \mathrm{a}$ & $25.88 \mathrm{fg}$ & $33.62 a$ \\
\hline $\mathbf{F}_{4}$ & $7.14 \mathrm{a}-\mathrm{g}$ & $5.02 \mathrm{fg}$ & $6.08 \mathrm{bc}$ & $11.80 \mathrm{f}-\mathrm{i}$ & $12.78 \mathrm{e}-\mathrm{h}$ & $12.29 \mathrm{e}-\mathrm{f}$ & $37.76 a-d$ & $28.38 \mathrm{~d}-\mathrm{g}$ & 33.07a \\
\hline $\mathbf{F}_{5}$ & $8.13 \mathrm{a}-\mathrm{d}$ & $5.96 \mathrm{~d}-\mathrm{g}$ & 7.05 a-c & $13.53 \mathrm{~d}-\mathrm{g}$ & $14.49 \mathrm{c}-\mathrm{f}$ & 14.01 de & $37.62 \mathrm{a}-\mathrm{d}$ & $28.93 \mathrm{c}-\mathrm{g}$ & 33.28a \\
\hline $\mathrm{F}_{6}$ & $8.27 \mathrm{a}-\mathrm{d}$ & $6.03 \mathrm{~d}-\mathrm{g}$ & 7.15 a-c & $12.86 \mathrm{e}-\mathrm{h}$ & $14.05 \mathrm{c}-\mathrm{g}$ & 13.46 ef & $39.29 a-c$ & $29.80 \mathrm{~b}-\mathrm{g}$ & $34.54 a$ \\
\hline$\overline{F_{7}}$ & $8.03 \mathrm{a}-\mathrm{d}$ & $6.11 \mathrm{~d}-\mathrm{g}$ & 7.07 a-c & $14.17 \mathrm{c}-\mathrm{g}$ & $17.09 \mathrm{bc}$ & $15.63 \mathrm{~cd}$ & $36.23 a-e$ & $26.20 \mathrm{e}-\mathrm{g}$ & 31.22a \\
\hline $\mathrm{F}_{8}$ & 8.39 a-d & $6.46 \mathrm{c}-\mathrm{g}$ & $7.43 \mathrm{ab}$ & $13.76 \mathrm{c}-\mathrm{g}$ & $19.85 \mathrm{ab}$ & $16.81 \mathrm{bc}$ & $37.79 a-d$ & $24.50 \mathrm{~g}$ & $31.14 a$ \\
\hline$F_{9}$ & $8.86 \mathrm{a}-\mathrm{c}$ & $7.04 \mathrm{a}-\mathrm{g}$ & $7.95 \mathrm{a}$ & $14.05 \mathrm{c}-\mathrm{g}$ & $21.37 \mathrm{a}$ & $17.71 \mathrm{a}-\mathrm{c}$ & $38.72 \mathrm{a}-\mathrm{d}$ & $24.71 \mathrm{~g}$ & $31.72 \mathrm{a}$ \\
\hline $\mathbf{F}_{10}$ & $9.03 \mathrm{ab}$ & $7.35 \mathrm{a}-\mathrm{f}$ & $8.19 \mathrm{a}$ & $14.59 \mathrm{c}-\mathrm{f}$ & $22.35 \mathrm{a}$ & $18.47 \mathrm{ab}$ & $38.24 a-d$ & $24.71 \mathrm{~g}$ & 31.48a \\
\hline$F_{11}$ & $9.24 \mathrm{ab}$ & $7.21 \mathrm{a}-\mathrm{f}$ & $8.23 \mathrm{a}$ & $16.67 \mathrm{~cd}$ & $21.12 \mathrm{a}$ & $18.89 \mathrm{ab}$ & $35.84 a-f$ & $25.42 \mathrm{~g}$ & $30.63 a$ \\
\hline$F_{12}$ & $9.43 \mathrm{a}$ & $7.51 \mathrm{a}-\mathrm{e}$ & $8.47 \mathrm{a}$ & $15.87 \mathrm{c}-\mathrm{e}$ & $22.60 \mathrm{a}$ & 19.23 a & $37.41 \mathrm{a}-\mathrm{d}$ & $24.84 f g$ & 31.12a \\
\hline Mean & 8.07 a & $6.13 \mathrm{~b}$ & & $13.11 \mathrm{~b}$ & $17.01 \mathrm{a}$ & & $38.43 \mathrm{a}$ & $26.76 \mathrm{~b}$ & \\
\hline $\begin{array}{c}\text { comparing } \\
\text { means of }\end{array}$ & $\begin{array}{c}\text { Genotypes } \\
\text { (G) }\end{array}$ & $\begin{array}{l}\text { Fertilizer } \\
\text { levels (F) }\end{array}$ & $\mathbf{G} \times \mathbf{F}$ & $\begin{array}{c}\text { Genotypes } \\
\text { (G) }\end{array}$ & $\begin{array}{l}\text { Fertilizer } \\
\text { levels (F) }\end{array}$ & $\mathbf{G} \times \mathbf{F}$ & $\begin{array}{c}\text { Genotypes } \\
\text { (G) }\end{array}$ & $\begin{array}{l}\text { Fertilizer } \\
\text { levels (F) }\end{array}$ & $\mathbf{G} \times \mathbf{F}$ \\
\hline S.Em. \pm & 0.164 & 0.616 & 0.871 & 0.384 & 0.888 & 1.256 & 0.493 & 2.370 & 3.352 \\
\hline
\end{tabular}

\begin{tabular}{l} 
G: Genotypes \\
\hline $\mathrm{G}_{1}: \mathrm{IC}-79147$ \\
\hline $\mathrm{G}_{2}:$ PRB-1 \\
\hline \\
\hline
\end{tabular}

F: Fertilizer levels $\left(\mathrm{kg} \mathrm{ha}^{-1}\right)$

\begin{tabular}{|c|c|}
\hline $\mathbf{F}_{1}: \mathrm{N}_{1} \mathrm{P}_{1} \mathrm{~K}_{1}(20: 10: 0)$ & $\mathbf{F}_{7}: \mathrm{N}_{2} \mathrm{P}_{2} \mathrm{~K}_{1}(40: 20: 0)$ \\
\hline $\mathbf{F}_{2}: \mathrm{N}_{1} \mathrm{P}_{1} \mathrm{~K}_{2}(20: 10: 10$ & $\mathbf{F}_{\mathbf{8}}: \mathrm{N}_{2} \mathrm{P}_{2} \mathrm{~K}_{2}(40: 20: 10)$ \\
\hline $\mathbf{F}_{3}: \mathrm{N}_{1} \mathrm{P}_{2} \mathrm{~K}_{1}(20: 20: 0)$ & $\mathbf{F}_{\mathbf{9}}: \mathrm{N}_{3} \mathrm{P}_{1} \mathrm{~K}_{1}(60: 10: 0)$ \\
\hline $\mathbf{F}_{4}: \mathrm{N}_{1} \mathrm{P}_{2} \mathrm{~K}_{2}(20: 20: 10)$ & $\mathbf{F}_{10}: \mathrm{N}_{3} \mathrm{P}_{1} \mathrm{~K}_{2}(60: 10: 10)$ \\
\hline $\mathbf{F}_{5}: \mathrm{N}_{2} \mathrm{P}_{1} \mathrm{~K}_{1}(40: 10: 0)$ & $\mathbf{F}_{11}: \mathrm{N}_{3} \mathrm{P}_{2} \mathrm{~K}_{1}(60: 20: 0)$ \\
\hline $\mathbf{F}_{6}: \mathrm{N}_{2} \mathrm{P}_{1} \mathrm{~K}_{2}(40: 10: 10)$ & $\mathbf{F}_{12}: \mathrm{N}_{3} \mathrm{P}_{2} \mathrm{~K}_{2}(60: 20: 10)$ \\
\hline
\end{tabular}


Among the fertility levels, application of 60: 20: $10 \mathrm{~N}: \mathrm{P}_{2} \mathrm{O}_{5}: \mathrm{K}_{2} \mathrm{O} \mathrm{kg} \mathrm{ha}{ }^{-1}$ was recorded significantly higher seed and straw yield (8.47 and $19.23 \mathrm{q} \mathrm{ha}^{-1}$ ), which was found on par with $F_{11}, F_{10}$ and $F_{9}$, whereas harvest index did not differ significantly with respect to fertility levels.

Among the interaction effects between genotypes and fertility levels, the genotype IC-79147 and PRB-1 along with application of 60: 20: $10 \mathrm{~N}: \mathrm{P}_{2} \mathrm{O}_{5}: \mathrm{K}_{2} \mathrm{O} \mathrm{kg} \mathrm{ha}{ }^{-1}$ recorded significantly higher seed and straw yield (9.43 and $22.60 \mathrm{q} \mathrm{ha}^{-1}$ respectively) and which were on par with $\mathrm{G}_{1} \times \mathrm{F}_{11}, \mathrm{G}_{1} \times \mathrm{F}_{10}, \mathrm{G}_{1} \times \mathrm{F}_{9}$ and $\mathrm{G}_{2} \times \mathrm{F}_{11}, \mathrm{G}_{2} \times \mathrm{F}_{10}, \mathrm{G}_{2} \times \mathrm{F}_{9}$ interactions for seed and straw yield respectively. The lower seed and straw yield were recorded with PRB-1 and IC-79147 genotypes with fertility level of 20: 10: $0 \mathrm{~N}: \mathrm{P}_{2} \mathrm{O}_{5}: \mathrm{K}_{2} \mathrm{O} \mathrm{kg} \mathrm{ha}{ }^{-1}$ (4.75 and 9.33 $\mathrm{q} \mathrm{ha}^{-1}$ respectively). Higher harvest index was recorded with IC-79147 with application of 20: 20: $0 \mathrm{~N}: \mathrm{P}_{2} \mathrm{O}_{5}: \mathrm{K}_{2} \mathrm{O} \mathrm{kg} \mathrm{ha}^{-1}$ (41.35) as compared to the other interactions.

The higher seed yield may be due to higher yield attributes viz., number of seeds per plant, seeds per clusters and test weight and translocation and accumulation of the photosynthates to economic part and genetic characteristic of particular genotype (IC79147). Higher straw yield with PRB-1 genotype was due to the higher growth parameters namely, plant height, branches per plant, leaf area, leaf area index and total dry matter production per plant. Similar results were reported by Saini and Negi (1998), Hulihalli and Shanthaveerayya (2017).

Based on the results obtained, it is concluded that genotype IC-79147 is very well suited to Northern Transition Zone of Karnataka with suitable cultural practices.

Application of 60: 20: $10 \mathrm{~kg} \mathrm{~N}: \mathrm{P}_{2} \mathrm{O}_{5}: \mathrm{K}_{2} \mathrm{O}$ ha

1 fertilizer to IC-79147 genotype is suitable for obtaining optimum seed yield $(9.43 \mathrm{q}$ ha ${ }^{1}$ ), with higher net returns ( $₹ 30,090 \mathrm{ha}^{-1}$ ) and $\mathrm{BC}$ ratio (3.11).

\section{References}

Christensen, K. B., Kaemper, M., Loges, R., Frette, R., Christensen, L. P. and Grevsen, K., 2007. Effects of nitrogen fertilization, harvest time, and species on the concentration of poly phenols in aerial parts and seeds of normal (Fagopyrum esculentum Moench) and tartary buckwheat (Fagopyrum taratricum L.). European J. Hort. Sci., 75 (4): 153-164.

Hongmei, L., Junsheng, B., Xia, L., Xiaoyan, D., Fang, S. and Rufa, L., 2003. The effects of fertilization on botanic characteristic and yield of tartary buckwheat (Fagopyrum tataricum L.). Proc. $9^{\text {th }}$ Int. Symp. on buckwheat. Advances in Buckwheat Res. 4. Cultivation and plant nutrition, 18-22, August, 2004, Cong. Cent., Univ. of Agric., Prague-Suchdol (Czech Republic), pp. 524-528.

Hulihalli, U. K. and Shantveerayya, 2017. Effect of planting geometry and nutrient levels on the productivity of buckwheat (Fagopyrum esculentum Moench). Int. J. Curr. Microbiol. App. Sci., 7 (2): 3369-3374.

Mahata, D., 2016. Studies on organic sources of nitrogen management in buckwheat (Fagopyrum esculentum Moench) and grain amaranth (Amaranthus hypochondriacus). Ph.D. Thesis, UKV Pundibhari, West Bengal (India), pp. 73.

Oshini, 2004. On the origin of cultivated buckwheat (Fagopyrum tarticum L.). Proc. $9^{\text {th }}$ Int. Symp. on buckwheat. Advances in Buckwheat Res. 4. Cultivation and plant nutrition, 18-22, August, 2004, Cong. Cent., Univ. of 
Agric., Prague-Suchdol (Czech Republic), pp. 16-21.

Saini, J. P. and Negi, S. C., 1998. Effect of spacing and nitrogen on Indian buckwheat (Fagopyrum esculentum Moench) under dry temperate condition. Indian J. Agron., 43: 351-354.

Sestak, Z., Castky, J. and Jarris, P. G., 1971. Plant analysis in production manual of methods (Ed. Jonk, W.), N. V. N. V. publications: 343-381.

Warchoowa, M., Kocon, A. and Mroczkowski, W., 1991. Response of buckwheat to different doses of nitrogen, potassium and magnesium, yield and mineral composition. Pamietnik puawski. 96: 23-35.

\section{How to cite this article:}

Maruti, U.K. Hulihalli and Aravind Kumar, B.N. 2018. Production Potential of Buckwheat (Fagopyrum esculentum Moench) as Influenced by Genotypes and Fertilizer Levels in Northern Transition Zone of Karnataka. Int.J.Curr.Microbiol.App.Sci. 7(09): 537-545. doi: https://doi.org/10.20546/ijcmas.2018.709.064 